WS5-B02

\title{
Direct VMS Targeting through Preserved Relative Amplitude Processed Seismic Imaging at Neves Corvo, Portugal
}

\author{
S. Yavuz* (Curtin University and DETCRC), J. Kinkela (Hiseis Pty Ltd), M. \\ Penney (Lundin Mining Corporation), V. Araujo (Lundin Mining \\ Corporation), R. Neto (Lundin Mining Corporation), A. Dzunic (Hiseis Pty \\ Ltd and Curtin University) \& M. Urosevic (Curtin University and DETCRC)
}

\section{SUMMARY}

Exploration of deep VMS deposits at the Neves Corvo mine on the Iberian Pyrite Belt was further advanced through a specialised application of surface seismic techniques. The contrast in impedances from elastic property measurements of core samples showed that there should be a significant difference in the seismic response of mineralisation compared to the surrounding host rocks. These results indicated that relative amplitude preservation processing may be of importance to help reduce the ambiguity in direct, seismic amplitude anomaly based targeting of Volcanogenic Massive Sulphides. However, such processing was not easy to implement due to an intrinsically low signal to noise ratio, complex 3D geology, high scattering level and often patchy and poor reflectivity. The newly obtained 3D seismic cube was calibrated with the existing boreholes to show superior ore zonation and precision required for the subsequent deep drilling campaign. 


\section{Amsterdam ' 14}

\section{Introduction}

Exploration and mining has been carried out for thousands of years on the Iberian Pyrite Belt (Strauss, 1977). In 2011 surface seismic methods were successfully applied to deep exploration at the giant Neves Corvo mine. However, direct mineral targeting from 3-D seismic data in mineralised terrains suffers from the problem of non-uniqueness, where different geology can result in similar amplitude responses (Taner, 1981). The objectives of this study were to mitigate the issue of non-uniqueness, delineate the major geological structures and to make direct targeting of Massive Sulphides (MS) from surface seismic data more reliable. This was achieved by first creating a database of the elastic property measurements of the major lithological units. Afterwards, through preserved relative amplitude (PRA) processing of the 3D seismic data, the reflectivity non-uniqueness issue was addressed to make direct targeting of mineral deposits from surface seismic data more reliable.

\section{Elastic Property Measurements}

Comprehensive elastic property measurements were carried out using ultrasonic pulse transmission tests at $1.0 \mathrm{MHz}$ for the 397 drill core samples from 13 different boreholes in 2012. Along with the standard cross-plots and graphs, the results were also collated by borehole, lithology and depth to understand the seismic signature of each main lithological unit.

The standard cross-plots showed that MS and stockwork (STWK) units were clearly discernible from the other rock units with their significantly higher densities and hence acoustic impedances. Another observation from these measurements was that the rhyolites did not provide a significant contrast with overlying silicates to generate a seismic reflection. MS are the highest impedance units relative to silicate units by a factor of about 2. However, the targets interpreted from the initial AGC processed seismic cube showed that in certain circumstances the rhyolite-silicate interface resulted in similar amplitude events to the MS. This indicated that seismic detectability is highly dependent on different factors and seismic data requires PRA processing.

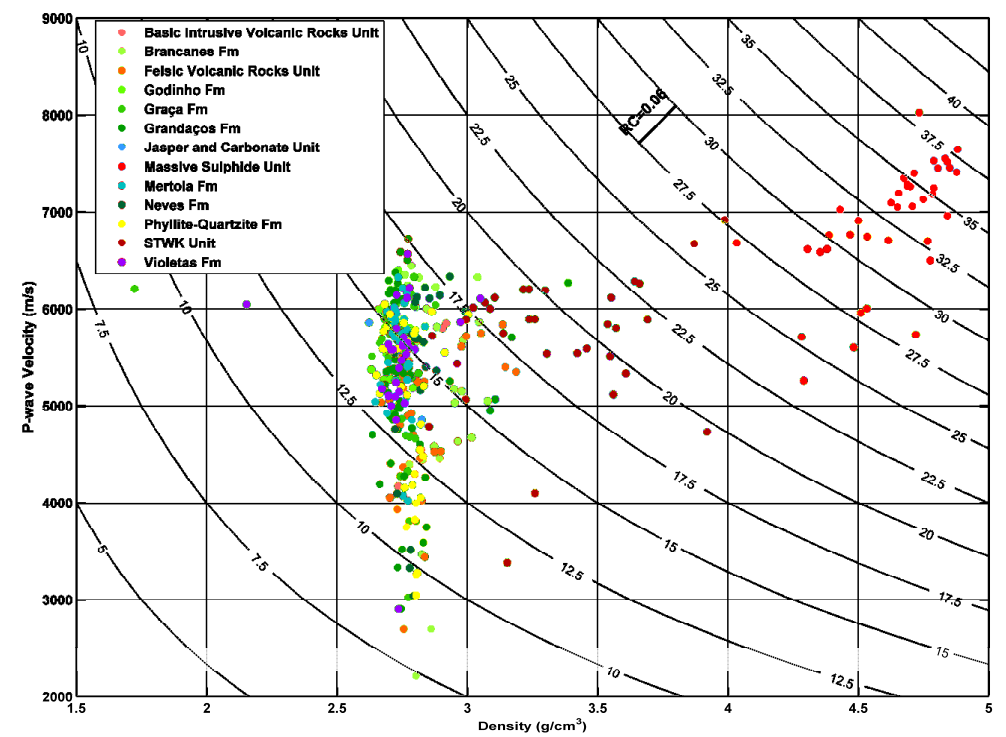

Figure 1 P-wave velocity and density cross-plot, collated by main lithological units. Constant acoustic impedance iso-contours (dashed-lines) are plotted at 0.06 reflection coefficient $(R C)$ intervals.

\section{Preserved Relative Amplitude Processing}

Analysis of the seismic response of ore bodies, such as massive sulphides, and direct targeting through seismic imaging requires strict PRA processing. In 2011, a basic seismic processing flow (AGC) was carried out on data acquired at Neves Corvo. In this study the $4.5 \mathrm{~km}^{2}$ sub data set focused on Semblana orebody reprocessed with PRA processing. Compared to AGC, PRA processing is more sensitive to noise. Trace killing, editing and muting was revised through several runs using different 


\section{Amsterdam''14}

signal analysis techniques to ensure that only traces with a signal/noise ratio above a certain threshold are passed for further processing. After shot domain ensemble balancing and time power gain application, surface consistent amplitude corrections were performed in an iterative manner. Also, the frequency spectrum of the data was preserved with the application of surface consistent spike deconvolution followed by a wide bandpass filter. The previous AGC processed version utilised a combination of DMO and post-stack migration. The new PRA processing applied an iterative prestack time migration. The final PRA processed seismic volume was found to be far superior to the AGC processed volume. While Semblana mineralisation was more easily discernable marker horizons (NC thrust and Culm) are also well defined in the PRA data set.

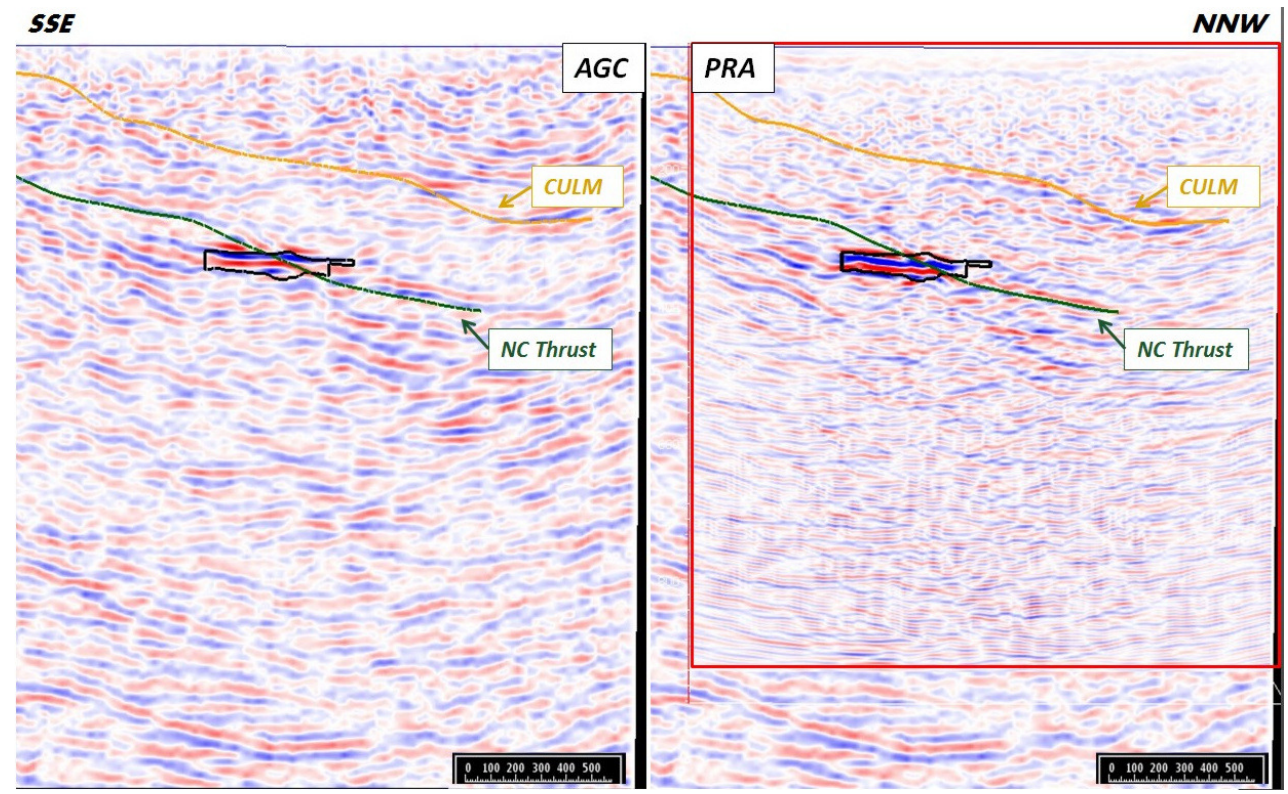

Figure 2 A seismic section from the new PRA Cube (inside the red rectangle on the right) overlain on a seismic section from the old AGC cube (on the left) and with the wireframes for the Semblana copper sulphide mineralisation (black outline), Neves Corvo Thrust and CULM.

\section{Conclusions}

Standard velocity-density cross-plots showed that MS and STWK units have the potential to provide large acoustic impedance contrasts when in contact with the host rock. Massive ore however has in most cases higher acoustic impedance than STWK indicating that PRA processing is the key to differentiating between massive ore and STWK formations. The comparison of AGC and PRA demonstrated the power of 3D seismic and also showed the significance of a careful; target oriented processing flow for ore delineation. Apart from the ore zone, marker horizons and major geological features are also well defined after PRA processing. Well ties are easily conducted with the PRA data set, which significantly increased the zonation and precision of targeting and enabled further work towards acoustic and elastic inversion.

\section{Acknowledgments}

We thank Lundin Mining Corporation and Hiseis Pty Ltd for providing data and continued support. The work has been supported by the Deep Exploration Technologies Cooperative Research Centre whose activities are funded by the Australian Government's Cooperative Research Centre Programme. We thank ASEG for its support to the project with a student research grant. We also thank Landmark Graphics, Paradigm and CGG for their software donations.

\section{References}

Strauss, G., Madel, J. and Alonso, F. F. [1977] Exploration Practice for Strata-Bound Volcanogenic Sulphide Deposits in the Spanish-Portuguese Pyrite Belt, Geology, Geophysics, and Geochemistry, Time-and Strata-Bound Ore Deposits. Springer, 55-93.

Taner, M.T. and Koehler, F. [1981] Surface consistent corrections. Geophysics, 46, 17-22. 